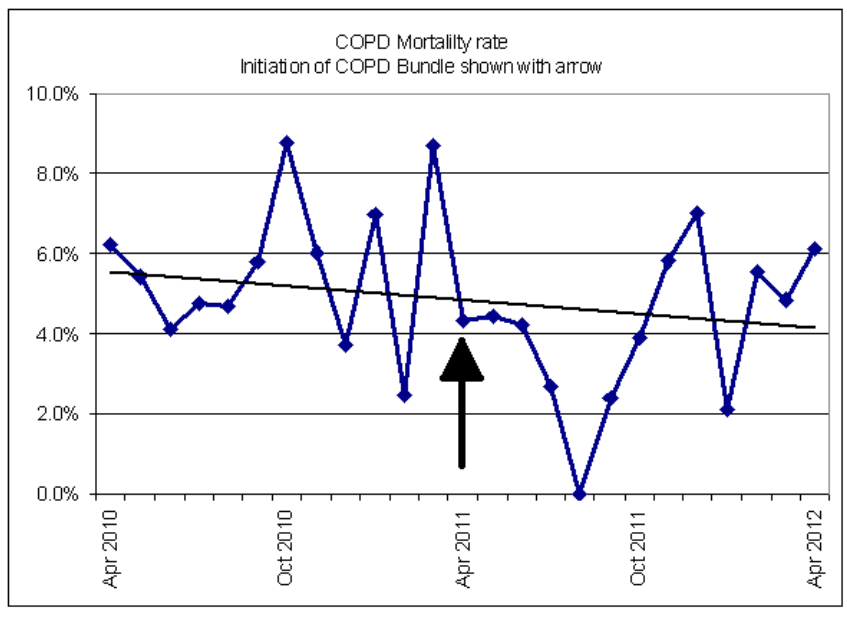

Abstract P290 Figure 1

\section{P291 COPD DISCHARGE BUNDLES: THE EXETER EXPERIENCE 2011-12}

doi:10.1136/thoraxjnl-2012-202678.383

DMG Halpin, P Batten, C Chamberlain. Royal Devon \& Exeter Hospital, Exeter, UK

Bundles which facilitate standardised quality care have been used for over 10 years (1). Recently, discharge bundles for COPD have been proposed as a way of improving outcomes, including reducing readmission rates, for patients discharged from hospital. We introduced one based on a version developed in London (2) in 2011. It comprised 5 items addressing self management, inhaler technique
(IT), smoking cessation advice (SA), pulmonary rehabilitation (PR), and follow up (FU). The bundle was completed by a respiratory nurse specialist (RNS) prior to discharge, they also planned to phone patients $24-72 \mathrm{hrs}$ post discharge.

The bundle was used in 67 patients discharged Oct 2011-Mar 2012. In total 324 patients with COPD were discharged during this time. Five patients were discharged before the bundle had been completed. It was not used if patients were confused, lived out of area, or went to intermediate or palliative care. The mean age was 72 (range 49-91), CAT score 22.5, MRC 3.66, FEV1 33\%, DOSE index 5.34 .

$58(87 \%)$ received written advice, $17(25 \%)$ were referred for or given SA, 65 (97\%) had IT checked, 21 (31\%) were referred for \& 19 (28\% declined) PR, 58(89\%) had FU requested. 39 (58\%) were phoned within 72 hrs and 60 (92\%) eventually. $77 \%$ of calls lasted 5 minutes or less. When contacted $37(57 \%)$ of patients said they were better and 52 (80\%) had no concerns. One patient was advised to restart steroids, 1 had a community matron visit arranged, 1 was readmitted \& 2 were given phone advice about coughs. One was constipated. Six of the 16 given SA were not smoking when phoned.

$12(18 \%)$ were readmitted within 30 days. This compares with a readmission rate of $27 \%$ in patients discharged during the same period who did not have the bundle completed.

Discharge bundles and follow up calls ensured this group of elderly patients with severe or very severe COPD had important aspects of their management assessed prior to discharge and this appeared to reduce readmission rates.

\section{References}

1. Burger CD, Resar RK Mayo Clinic Proceedings. 2006; 81(6):849-850

2. Hopkinson NS, et al. Thorax 2012; 67:90-2. 